Received 2021-02-27

Revised 2021-02-27

Accepted 2021-05-10

\title{
Concept Analysis of Recovery in Nursing
}

\author{
Azade Safa $^{1}$, Mohsen Adib-Hajbaghery ${ }^{1 凶}$
}

${ }^{1}$ Trauma Nursing Research Center, Kashan University of Medical Sciences, Kashan, Iran

\begin{abstract}
Recovery is an abstract multidimensional concept. There is no clear definition for recovery because this concept has specific meanings for individuals. This study aimed to analyze the concept of recovery in nursing. A concept analysis was conducted using Walker and Avant's method. An extensive literature review was conducted. The primary search key terms were "recovery," "concepts related to recovery," and "nursing." The publication date was limited to 2006-2019. Data related to the definitions, attributes, antecedents, and consequences of recovery were extracted from the reviewed studies. We found several attributes for this concept. Physical attributes of recovery included fighting with illness, experiencing an injury, and pain. Mental attributes were achieving self-restoration, hopefulness, self-confidence, self-identity, the meaning of life, and personal growth. Social attributes were self-determination, autonomy, support, social confidence, and active participation in social relationships. The antecedents and consequences of recovery were classified into physical, mental, and social dimensions. Model, borderline, and contrary cases were presented. Recovery is a complex and dynamic process, which is unique to individuals based on their characteristics and conditions.
\end{abstract}

[GMJ.2021;10:e2164] DOI:10.31661/gmi.v10i0.2164

Keywords: : Recovery; Nursing; Concept Analysis

\section{Introduction}

$\mathrm{T}$ The term recovery has appeared in medical literature since the late 1960s and early 1970s [1]. Initially, this term was used to protect the rights of patients with mental health problems. Later, the term was used in different fields of medicine and healthcare. Despite its long history, it has not been subject to enough academic research $[2,3]$.

The term recovery is used in different scientific disciplines. For instance, this term in computer science refers to the retrieval of data, and in sports science refers to the return of physical strength.

The term recovery stands for regaining

GMJ

Copyright $\odot$ 2021, Galen Medical Journal. This is an open-access article distributed under the terms of the Creative Commons Attribution 4.0 International License (http://creativecommons.org/licenses/by/4.0/) Email:info@gmj.ir physical and mental abilities in medical sciences and is used in multiple areas, including mental disorders, drug addiction, trauma, and surgery $[1,2,4-6]$.

In medicine and healthcare, recovery is considered to be a multidimensional concept with physical, mental, and social dimensions [6]. Recovery is a subjective and abstract concept, which seems to overlap with concepts such as wellbeing, healing, and rehabilitation $[2,7,8-10]$.

Because recovery is a subjective concept, it cannot be easily measured. There is a need for the identification of its attributes and boundaries to understand this concept.

Different definitions are suggested for the

\footnotetext{
Correspondence to:

Mohsen Adib-Hajbaghery, Trauma Nursing Research Center, Kashan University of Medical Sciences, Kashan, Iran. Telephone Number: +983155540021

Email Address: adib1344@yahoo.com
} 
term recovery. Some scholars defined recovery as returning to a normal state after a period of problems or illness [11].

Others in the field of mental health defined the term as a unique journey in human life or the ability to live a meaningful life, which can be associated with race, ethnicity, culture, and religion $[12,13]$.

There are differences in different races or ethnicities in acceptance and accessibility to health care, which affects their recovery after illness. Also, religion helps during the recovery process [12].

Recovery is also defined as a process of change, through which people improve their health, direct their lives, and achieve their full potential [2]. While some view recovery as an individual process, others believe that recovery can be achieved only through collaborating patients and/or clients with healthcare providers $[6,14]$.

The wide variety of definitions for recovery denotes the lack of consensus over its meaning. Nurses need to obtain an in-depth understanding of the term recovery in order to set realistic care-related goals and improve patient outcomes [15-19].

A lack of a clear definition for recovery can affect nurses' practice and prevent them from achieving goals regarding patients' health outcomes, for the reason that the lack of proper understanding of this concept will stop this process in the early stages. In this study, we analyzed the concept of recovery in nursing.

\section{Search Strategies}

This concept analysis was conducted in 2019 using Walker and Avant's method [20]. Concept analysis assesses the attributes of a concept and determines overlaps of the concept with other concepts in terms of their meaning.

Walker and Avant's method for concept analysis consists of eight steps: selecting a concept, identifying the aim of the analysis, recognizing the uses of the concept, determining the defining attributes of the concept, developing a model case, identifying borderline, related, and contrary cases, determining the antecedents and the consequences of the concept and defining the concept's empirical referents [20].

An extensive literature search was performed to retrieve relevant studies using different search engines, including ScienceDirect, PubMed, Scopus, and Google Scholar.

The literature search was performed in two steps. In the first step, search key terms were "recovery" OR its related concepts (i.e., rehabilitation, healing, wellbeing, illness management, and recovery measurement tools) AND "nursing" in title/abstract; and in the second step, the key terms "recovery" (title) AND "nursing" (title/abstract) were used.

Articles that were accessible in full-text, written in English, published between 2006 and 2019, addressed recovery or its related concepts (such as rehabilitation, healing, wellbeing, and illness management), and addressed recovery measurement tools were included. Moreover, the reference list of the retrieved articles and Google search engine were used to find other relevant documents.

After excluding duplicates, 39 documents-10 theoretical and 29 empirical articles-were included in the study (Figure-1). This study extracted definitions, attributes, antecedents, and consequences of recovery from the reviewed studies, and the data were analyzed inductively.

\section{Selecting a Concept}

Recovery is an abstract multidimensional concept that has not been clearly defined in health-related literature [6]. The concept has some similar attributes with concepts such as wellbeing, healing, and rehabilitation [8-10]. As an abstract concept, recovery and its dimensions cannot be measured using quantitative methods, and there is no tool for assessment of its dimensions. A concept analysis of recovery can help clarify this concept and facilitate its understanding and application in nursing education and practice.

\section{Determining the Aims of Analysis}

The purposes of this study were to determine the attributes, dimensions, antecedents, and consequences of recovery in nursing and to differentiate this concept from other related concepts [20]. 


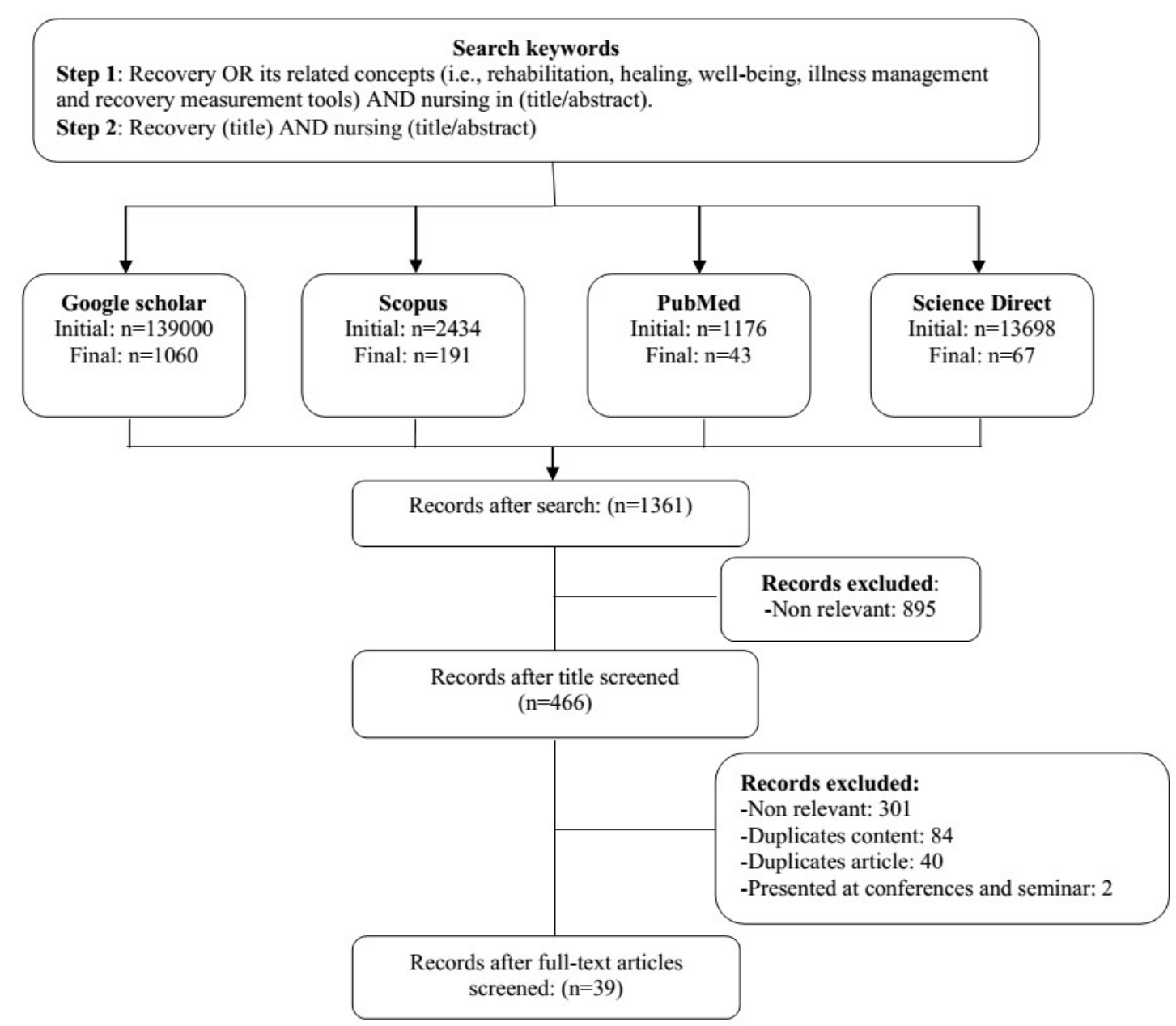

Figure 1. Flow diagram for study selection

\section{Results}

\section{The Definition and the Uses of Recovery}

Different definitions and uses have been suggested for the concept of recovery in different fields. Dictionaries propose general definitions, while scientific fields like nursing and psychology propose more field-specific definitions.

\subsection{Definition of Recovery in Dictionaries}

The Longman Dictionary of Contemporary English defines recovery as regaining health and returning to a normal state after a period of problems or health conditions or having a missing item returned [11].

The Oxford English Dictionary defines recovery as "a return to a normal state of health, mind, or strength" [21], and the Merriam-Webster dictionary defines it as the "process of combating a disorder or a real perceived problem" [22].

\subsection{Definitions and Uses of Recovery in} Nursing

Nurses often use the concept of recovery to describe an improvement in their clients' and patients' health. Most people experience illness, disability, injury, or trauma during their lives, and some may experience financial strains, voluntary or mandatory migration, natural disasters, and rapid social changes [2]. Recovery can be beyond eliminating the effects of illness and include the recovery of physical, mental, and social functioning [6]. A previous study reported that recovery consists of self-orientation, family orientation, social orientation, and illness orientation [23].

Recovery is a unique personal experience and includes both physical and mental components. 
In clinical practice, recovery is achieved when a person gets better from disease and does not experience any further symptoms, both physically and mentally [24]. Recovery of physical symptoms usually occurs faster than mental symptoms because people mainly focus on their physical symptoms than their mental issues [24, 25].

In the acute phase of an illness, the main focus is on treating the symptoms, while the focus in the convalescence phase is on regaining autonomy, improving feelings, and health [24]. Compared to the physical component of recovery, mental recovery is more time-consuming and requires changes in attitudes, values, feelings, aims, skills, roles, fulfillment of wishes, regaining self-confidence, and control over life [24-26].

Mental recovery is based on acknowledging illness-related disabilities and personal abilities and the development of new meanings and purposes in life to create a satisfactory and hopeful life [12, 27, 28]. Recovery of social functioning relies on rebuilding social confidence and regaining active participation in social relationships [5, 23]. Usually, recovery of physical symptoms occurs first, and mental and social problems later, but it may be different depending on the person and his/ her situation $[24,25]$.

Recovery is not an endpoint; instead, it is an ongoing process $[2,7]$. Patients describe this concept as a unique, personal, non-linear experience and journey [12]. Recovery relies on regaining hope, identity, connectedness, autonomy, support, and power.

Accordingly, recovery - especially mental and social recovery - is the key to enter a meaningful life $[13,29]$. The recovery process varies from person to person, as well as from illness to illness in terms of its duration and level. This difference can be associated with different factors, such as underlying conditions, personality type, race, and culture [13, $24,27,30,31]$. Recovery is a result of teamwork and mostly happens at home; therefore, several groups, such as healthcare providers, family members, friends, social networks, and people in the workplace, can play significant roles in the process of recovery [27].

The concept of recovery has also been broadly used in the Illness Management and Recovery (IMR) programs. IMR is a service that provides support to help patients manage their mental issues and move forward throughout their recovery process [32].

In IMR, recovery includes clinical and personal dimensions. Clinical recovery refers to a reduction in signs and symptoms of mental illnesses and restoration of cognitive, social, and occupational functioning. Personal recovery is defined as a process of constructing a personally meaningful life even though the individual experiences the limitations related to a mental illness [33].

The concept of recovery has overlaps and distinctions with other concepts, such as healing, wellbeing, and rehabilitation. These concepts are described and differentiated from the concept of recovery in the following paragraphs.

\subsubsection{Healing}

Healing refers to the physical reconstruction of the damaged cells and/or tissues after an injury and is affected by factors such as tissue perfusion and the type of injury.

However, recovery is a more comprehensive concept that comprises physical, mental, and social dimensions and can be improved by motivation and social support. However, healing can be considered as a primary step in the recovery process. Healing is related to physical health and functioning, which is a primary goal early in a post-injury period $[8$, 34].

\subsubsection{Rehabilitation}

Rehabilitation is a process for helping individuals regain their functional abilities after a crisis, illness, and injury [9]. In other words, rehabilitation can facilitate recovery by helping individuals cope with physical damages due to an illness and/or injury. Recovery is a broader concept compared to rehabilitation because recovery is not limited to restoring physical and social abilities. Recovery is the ability to perform activities while improving self-confidence, enjoyment, control over life, and a positive feeling about self [9].

\subsubsection{Wellbeing}

Wellbeing is a state of feeling comfortable, healthy, and happy $[10,35]$ and is considered the ultimate goal in the process of recovery. 
Taking all the statements into account, recovery is both an outcome and a process wherein individuals strive to achieve their goals $[10,36]$.

\section{Defining Attributes of Recovery}

Abstract concepts have more than one defining attribute. In concept analysis, the most appropriate defining attributes of a target concept are examined [20]. The attributes of the concept of recovery can be classified into physical, mental, and social dimensions.

Physical attributes include fighting with an illness, having a present or past injury and pain, and trying to manage physical problems caused by illnesses or injuries $[4,9,37]$.

Mental attributes include attempting to reattain a feeling of self-efficacy [32, 38], restoring self, finding hope, regaining self-confidence, admiring self, discovering one's identity, remaining optimistic about the future, and having positive feelings about personal growth, and giving meaning to life [1, $2,6,13,28]$.

Social attributes include attempting to achieve self-determination, autonomy, support, rebuilding social confidence, regaining the ability to actively participate in social relationships, regaining control over life, attaining goals, developing skills, and modifying lifestyle in a continuous non-linear unique way that can be accomplished with collaboration with others $[5,7,27]$.

\section{Antecedents of Recovery}

Antecedents of a concept are events and conditions that should be present before its occurrence [20]. Recovery is a dynamic process that necessitates an active involvement of the patient and recognition of a need for recovery [27]. Based on the physical, mental, and social attributes of recovery, the concept's antecedents can also be categorized into physical, mental, and social antecedents.

\subsection{Physical Antecedents of Recovery}

Factors such as injury, trauma, disorder, illness, reduced physical energy, pain, and disability can affect physical health and recovery. The higher the intensity of these factors, the slower the recovery rate.

Accordingly, because of health declines among older adults, their recovery from physical issues is more challenging compared to younger people [39]. Evidence indicated diversities among racial populations in terms of health status and recovery [40]. The main physical antecedents of recovery include declined physical health, physical capacity, and recognizing a need for improving physical health $[5,41]$.

\subsection{Mental Antecedents of Recovery}

Loss of important individuals and belongings, grief, worry, uncertainty, reduced security and mental energy, mental fatigue, hopelessness [29, 32], emotional disorders, stress, despair, notoriety, altered self-image, and reduced self-confidence can negatively affect mental health $[6,24,26]$. Loss of beloved ones is non-modifiable; however, hopefulness toward the future can help the person in terms of recovery from the loss [25].

Several mental antecedents of recovery are related to individuals' perceived needs, including a need for feeling better, feeling of development and promotion, acknowledging personal limitations and impaired health, recognizing personal abilities and strengths, coping strategies, and a power to fight and overcome life challenges [27, 28]. In this regard, providing support and education about the individual's potentials and capabilities can help the individual in terms of recovery from illnesses or crises.

\subsection{Social Antecedents of Recovery}

Recovery can be affected by social factors such as socioeconomic status, low education level, sociocultural and population changes, migration, dependence on others, and natural disasters $[5,23]$. The other social antecedents of recovery are social support, participatory approaches, and the need to change social relationships. These factors can reduce stress and create a sense of safety and security $[19$, 29].

\section{Consequences of Recovery}

Consequences of a concept are those events or incidents that occur due to the occurrence of the concept [20]. Recovery has physical, mental, and social consequences. 


\subsection{Physical Consequences of Recovery}

The main physical consequences of recovery are lifestyle modification, physical health improvement, pain management, relapse prevention, feeling energetic, physical symptoms remission, mobility improvement, regaining previous physical abilities, and acquiring new physical abilities [4, 29, 38, 42, 43].

\subsection{Mental Consequences of Recovery}

Mental consequences of recovery include improving illness self-management, mental restoration, satisfaction, happiness, coping behaviors, and feelings about self. Moreover, the patient may construct a new self or revive the past self $[1,44]$.

The other mental consequences of recovery include positive changes in attitudes, roles, and identity, and also hopefulness, control over life, positive self-concept, self-confidence, self-belief, growth and development, goal setting, and goal attainment.

The ultimate mental consequences of recovery are improved quality of life, self-admiration, and a meaningful and satisfactory life $[1,6$, 23, 28, 29, 32, 38].

\subsection{Social Consequences of Recovery}

Social consequences of recovery include active participation in social activities, increased social relationships [37], reduced social isolation [29], improved autonomy [1, 27], enhanced social identity [28, 45], and improved participation in the community [37]. A model case represents an example of the use of the concept to clarify its attributes and help readers understand the concept [20].

\section{Model Case}

Mr. A, a 65-year-old driver with a sedentary lifestyle, was referred to the cardiac emergency room with diaphoresis and chest pain. $\mathrm{He}$ had experienced the symptoms while driving. After a primary assessment, he underwent an emergency coronary artery bypass graft surgery and was discharged from the hospital one week later. After discharge, he felt tired and short of breath while walking. He also felt burning and pain in his sutures, and his appetite was decreased due to changes in his diet after the surgery.

Furthermore, based on the doctor's advice for decreasing the infection risk after surgery, his relationships with friends and colleagues were reduced. He was worried about the recurrence of symptoms and his ability to return to work. $\mathrm{He}$ received cardiac rehabilitation services. $\mathrm{He}$ also received information about his diet, physical activity, and stress management from a specialist nurse.

Currently, three months after his surgery, his surgical site is healing, and his appetite has improved. He also adheres to his diet, has a regular sleep pattern, and walks for half an hour every day. He reports no difficulty during physical activities and helps his wife with household chores. Also, he can drive and has returned to his job. He goes hiking with his family once a week.

The attributes of recovery, in this case, include attempts to cope with the conditions, develop self-control and a healthy lifestyle, and regain health.

The antecedents of recovery are fighting against disease, worry, uncertainty, and concern about the future, decreased appetite, disturbed sleep, poor functioning, reduced social activities, and a need to regain health and return to the normal life. The consequences of recovery include accepting and adhering to the diet and exercise, stress management, returning to work, active participation in social activities, and self-empowerment.

\section{Borderline Case}

A borderline case is an example that represents most of, not all, the defining attributes of the concept [20].

Mrs. B is a 50-years-old woman with type II diabetes. Recently, she was hospitalized in the medical ward of a hospital for treatment of a diabetic foot ulcer in her big toe that had developed over two weeks. Her fasting blood sugar was above $300 \mathrm{mg} / \mathrm{dL}$. She takes her medications properly. She uses a glucometer to monitor her blood sugar once a day.

However, she has irregular physical activity and does not adhere to a diabetic diet. She was treated and trained by a wound care nurse. Despite medical efforts and care for her foot ulcer, her wound did not heal significantly. The physician told her that her toe might be amputated if the wound did not heal.

The attribute of recovery, in this case, is a 
moderate level of efforts to manage the disease. The antecedents are reduced health and the need for wound healing. The consequence is minimal wound healing.

\section{Contrary Case}

The contrary case fulfills none of the defining attributes of the concept [20].

Mrs. C is a 30 -year-old single teacher. One month ago, she experienced head trauma in a car accident and was hospitalized in an intensive care unit with a Glasgow Coma Scale (GCS) score of 7. Despite medical treatments and nursing care, her health condition declined, and her GCS score decreased to 3. Her pupils became unresponsive to light, and she needed mechanical ventilation to receive ventilation using the synchronized intermittent mandatory ventilation mode. The patient's family was disappointed about her recovery due to the severity of her injuries.

In this case, the attributes of recovery are not present. The antecedents include declined health, reduced consciousness, and medical efforts to treat the patient. Because there is a deteriorating health condition, and recovery is not present, there is no consequence associated with recovery in this case.

\section{Empirical Referents}

Empirical referents are the detectable characteristics of a concept, which indicate the presence of the concept. The main aim of defining the empirical referents is to facilitate the concept measurement [20]. According to Walker and Avant [20], abstract concepts have abstract defining attributes; therefore, finding and defining their empirical referents are challenging. There are different tools for recovery measurement. Tools for measuring quality of life, pain intensity, and activities of daily living can measure some aspects of recovery. For recovery assessment using these tools, individuals are asked to rate improvements in their conditions over some time [41, 42, 46, 47].Moreover, tools such as the Recovery Knowledge Inventory and the Recovery Attitudes Questionnaire help assess knowledge and attitudes about recovery [48]. Recovery cannot be measured using a single tool because of its abstract attributes and its different contributing factors.

\section{Discussion}

In this study, we examined the concept of recovery in nursing. Our findings showed that recovery is a complex, dynamic, and evolutionary process that is unique to each person and includes physical, mental, and social dimensions.

The process of recovery occurs through close collaboration among the individual, family members, and healthcare providers. One of the primary responsibilities of nurses in this process is to facilitate collaboration among these individuals. The current study presented physical, mental, and defining social attributes, antecedents, and consequences of the concept (Figure-2).

Recovery is a process of fighting or coping with an illness, which can regain physical, mental, and social health, feeling hope, regaining self-confidence, and developing empowerment. Understanding the concept of recovery can help nurses identify patients' physical, mental, and social capacities. Based on the identified capacities, nurses can develop a care plan to help patients set realistic goals for improving their health, autonomy, and social interactions. Achieving these goals can help individuals fulfill a meaningful and satisfactory life.

Previous studies on the concept of recovery mainly addressed clinical recoveries — such as recovery from trauma or surgery - or recovery from mental disorders and addiction [1, $2,4,5]$. These studies mostly addressed the physical and mental dimensions of recovery $[2,4,15]$. Moreover, recovery in previous studies was considered as restoration, illness alleviation, and returning to a normal state. Our results showed that recovery is a more comprehensive concept and can develop new abilities and higher levels of functioning.

Although the concept of recovery seems to overlap with other concepts, such as wellbeing, healing, and rehabilitation, the present study results revealed fundamental differences among these concepts. Healing focuses on the physical reconstruction of damaged cells and tissues after an injury $[8,34]$.

Rehabilitation is the process of coping with physical changes associated with illness and/ 


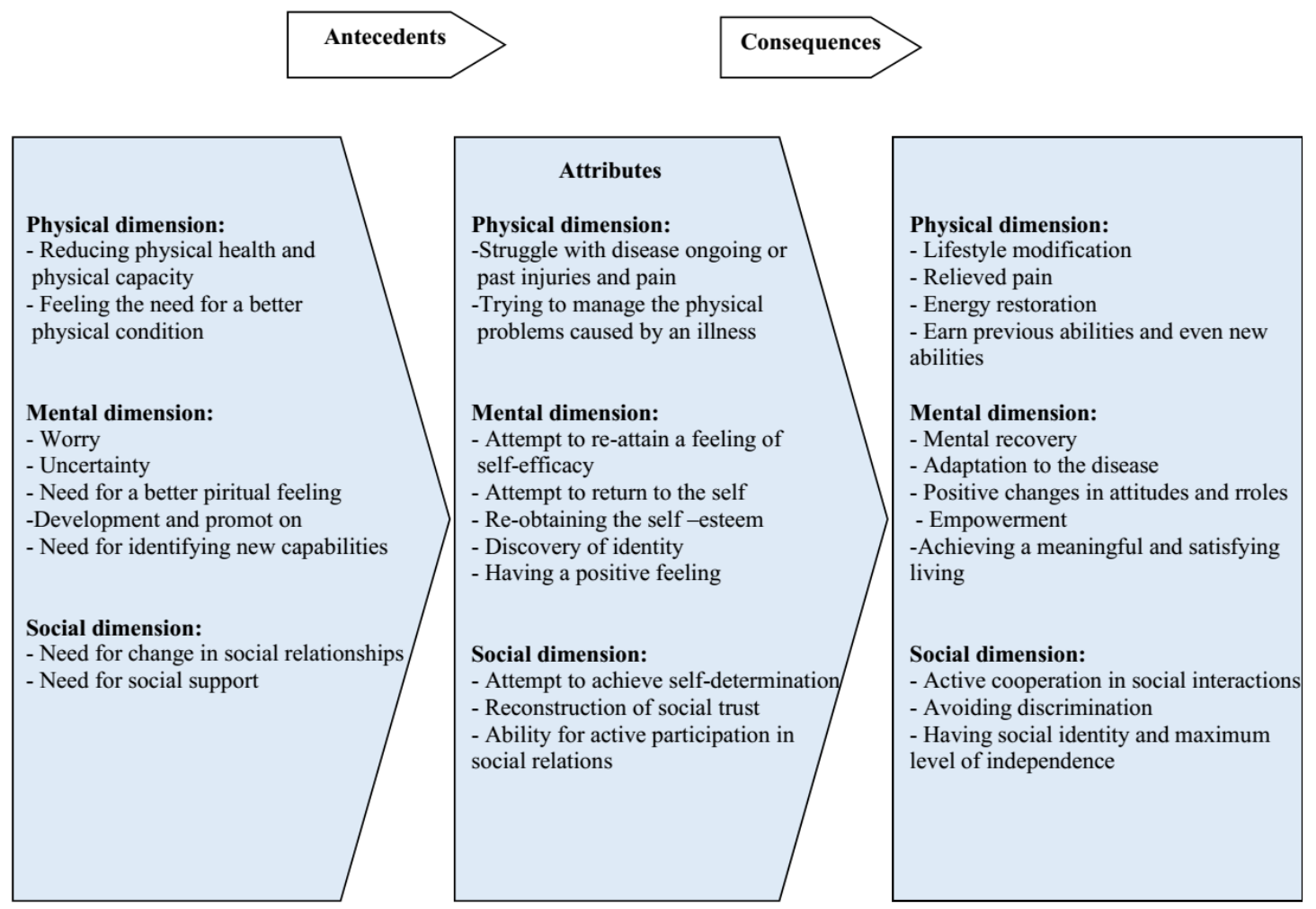

Figure 2. A schematic model for analysis concept of recovery

or injury to regain functional abilities [9]. Wellbeing is a goal in the process of recovery. Wellbeing is not only an ultimate outcome that an individual strives to achieve, but it is part of a continuing process that helps people to attain their goals $[10,36]$. Accordingly, the process of recovery from illness can begin with healing and ends in wellbeing.

Recovery is necessary for human growth and development after illnesses, traumas, or other conditions that reduce energy or strength. Nurses need to guide patients in the process of recovery accurately.

Although recovery can take a person to the previous level of abilities or even to a point beyond it, nurses and patients frequently restrict recovery goals to a limited level. Recovery is a creative and participatory process that can improve individuals from despair to hope, weakness to strength, and isolation to social interactions.

The goal in this process is growth and development. Through recovery, patients can regain their abilities, develop their capacities and strengths $[14,28]$, and achieve a meaningful and satisfactory life.

\section{Conclusion}

This concept analysis showed that the concept of recovery includes physical, mental, and social dimensions. Recovery is a complex, dynamic, and evolutionary process that is unique to each individual.

The main attributes of recovery include fighting with an illness, managing physical problems, attempting to reattain a feeling of self-efficacy, remaining optimistic about the future, giving meaning to life, attempting to achieve self-determination, and participating in social interactions. The main antecedents of recovery include reduced physical health, a perceived need for a better physical and spiritual condition, social support and collaborative approaches, identifying the individuals' potentials and capabilities, and a change in social relationships. Successful recovery can result in lifestyle modification, regaining 
abilities, social identity, and hope, achieving a meaningful and satisfying life, and experiencing an optimal level of independence.

Nurses can address the attributes, antecedents, and consequences of recovery to perform accurate patient assessments, develop care plans, and evaluate the outcomes of their plans.

\section{Conflict of Interest}

There are no conflicts of interest.

\section{References}

1. McCauley CO, McKenna HP, Keeney S, McLaughlin DF. Concept analysis of recovery in mental illness in young adulthood. J Psychiatr Ment Health Nurs. 2015; 22(8): 579-89.

2. Laudet $A B$. What does recovery mean to you?. Lessons from the recovery experience for research and practice. $\mathrm{J}$ Subst Abuse Treat. 2007; 33(3): 243-56.

3. Petros R, Solomon P, Linz S, DeCesaris M, Hanrahan NP. Autovideography: The Lived Experience of Recovery for Adults with Serious Mental Illness. Psychiatr Q. 2016; 87(3): 417-26.

4. Jakobsson J, Idvall E, Kumlien C. The lived experience of recovery during the first 6 months after colorectal cancer surgery. J Clin Nurs. 2017; 26(23-24): 4498-505.

5. Nasirian S, Fagevik Olsén M, Engström M. Patients' Experiences of Their Recovery Process after Minor Physical Trauma. J Trauma Nurs. 2018; 25(4): 233-41.

6. Baccari IO, Campos RT, Stefanello S. Recovery: revisão sistemática de um conceito [Recovery: systematic review of a concept]. Cien Saude Colet. 2015; 20(1): 125-36.

7. Castillo H, Ramon S, Morant N. A recovery journey for people with personality disorder. Int J Soc Psychiatry. 2013; 59(3): 264-73.

8. Ardolino A. Healing and Recovery: Is there a difference?. Internet J Surg. 2006; 13(1): 1-5.

9. Claydon JH, Robinson L, Aldridge SE. Patients' perceptions of repair, rehabilitation and recovery after major orthopaedic trauma: a qualitative study. Physiotherapy. 2017; 103(3): 322-9.
10. Wiltgen A, Shepard C, Smith R, Fowler JC. Emotional rigidity negatively impacts remission from anxiety and recovery of well-being. J Affect Disord. 2018; 236: 69-74.

11. Richards JC, Schmidt RW. Longman dictionary of language teaching and applied linguistics. 4th ed. Routledge; 2013.

12. Al-Omari H, Hamed R, Abu Tariah H. The Role of Religion in the Recovery from Alcohol and Substance Abuse among Jordanian Adults. J Relig Health. 2015; 54(4):1268-77.

13. Leamy M, Bird V, Le Boutillier C, Williams J, Slade M. Conceptual framework for personal recovery in mental health: systematic review and narrative synthesis. Br J Psychiatry. 2011; 199(6): 445-52.

14. doseć S, Morovic S, Basic Kes V, Zavoreo I, Jurasic M-J, Demarin V. Enhancement of stroke recovery by music. Period Biol. 2012; 114: 397-401.

15. Burch J. Enhanced recovery, stomas and the community nurse. Br J Community Nurs. 2013; 18(5): 214-20.

16. Jones C. Recovery post ICU. Intensive Crit Care Nurs. 2014; 30(5): 239-45.

17. Lee J, Seo E, Choi J, Min J. Effects of patient participation in the management of daily nursing goals on function recovery and resilience in surgical patients. J Clin Nurs. 2018; 27(13-14): 2795-03.

18. Maddigan J, LeDrew K, Hogan K, Le Navenec CL. Challenges to recovery following early psychosis: Nursing implications of recovery rate and timing. Arch Psychiatr Nurs. 2018; 32(6): 83644. 
19. Waks S, Scanlan JN, Berry B, Schweizer R, Hancock N, Honey A. Outcomes identified and prioritised by consumers of Partners in Recovery: a consumer-led study. BMC Psychiatry. 2017; 17(1): 338.

20. Walker LO, Avant KC. Strategies for theory construction in nursing. 6th ed. Upper Saddle River, NJ: Pearson Prentice Hall; 2005.

21. Stevenson A. Oxford dictionary of English. 3th ed. Oxford University Press, USA; 2010.

22. Merriam-Webster Online Dictionary. (2019). Retrieved from http://www. merriam-webster.com/dictionary/ recovery (accessed May 18, 2019).

23. Jose D, Ramachandra, Lalitha K, Gandhi S, Desai G, Nagarajaiah. Consumer perspectives on the concept of recovery in schizophrenia: A systematic review. Asian J Psychiatr. 2015;14:13-8.

24. Lofthus AM, Westerlund H, Bjørgen D, et al. Recovery concept in a Norwegian setting to be examined by the assertive community treatment model and mixed methods. Int J Ment Health Nurs. 2018; 27(1): 147-57.

25. Fecher-Jones I, Taylor C. Lived experience, enhanced recovery and laparoscopic colonic resection. Br J Nurs. 2015; 24(4): 223-28.

26. Warner R. Recovery from schizophrenia and the recovery model. Curr Opin Psychiatry. 2009; 22(4): 374-80.

27. Davis I, Alexander D. Recovery from disaster. Routledge; 2015.

28. Piltch CA. The role of self-determination in mental health recovery. Psychiatr Rehabil J. 2016; 39(1): 77-80.

29. Beentjes TAA, van Gaal BGI, Goossens PJJ, Schoonhoven L. Development of an e-supported illness management and recovery programme for consumers with severe mental illness using intervention mapping, and design of an early cluster randomized controlled trial. BMC Health Serv Res. 2016; 16:20.

30. El-Guebaly N. The meanings of recovery from addiction: evolution and promises. $\mathrm{J}$ Addict Med. 2012; 6(1): 1-9.

31. Ioannou L, Cameron PA, Gibson SJ, et al. Financial and recovery worry one year after traumatic injury: A prognostic, registry-based cohort study. Injury. 2018; 49(5): 990-1000.

32. Mueser KT, Meyer PS, Penn DL, Clancy R, Clancy DM, Salyers MP. The Illness Management and Recovery program: rationale, development, and preliminary findings. Schizophr Bull. 2006;32:32-43.

33. Dalum HS. Illness Management and Recover- Patient Outcomes and Staff Perspectives. Schizophr Res. 2007; 96: 232-45.

34. Wang, PH, Huang BS, Horng HC, Yeh CC, Chen YJ. Wound healing. J Chin Med Assoc. 2018; 81(2): 94-101.

35. Keeman A, Näswall K, Malinen S, Kuntz J. Employee Wellbeing: Evaluating a Wellbeing Intervention in Two Settings. Front Psychol. 2017; 8:505.

36. Diener E. The science of well-being: The collected works of Ed Diener. Dordrecht: Springer; 2009.

37. Tan CHS, Ishak RB, Lim TXG, Marimuthusamy P, Kaurss K, Leong JJ. Illness management and recovery program for mental health problems: reducing symptoms and increasing social functioning. J Clin Nurs. 2017; 26(2122): 3471-85.

38. Sit JW, Chair SY, Choi KC, et al. Do empowered stroke patients perform better at self-management and functional recovery after a stroke?. A randomized controlled trial. Clin Interv Aging. 2016; 11: 1441-50.

39. Deiner S, Silverstein JH. Long-term outcomes in elderly surgical patients. Mt Sinai J Med. 2012; 79(1): 95-106.

40. van de Rijt A, Song HG, Shor E, Burroway R. Racial and gender differences in missing children's recovery chances. PLoS One. 2018; 13(12): e0207742.

41. Yazdi-Ravandi S, Taslimi Z, Jamshidian N, Saberi H, Shams J, Haghparast A. Prediction of Quality of life by SelfEfficacy, Pain Intensity and Pain Duration in Patient with Pain Disorders. Basic Clin Neurosci. 2013; 4(2): 117-24.

42. Bowyer AJ, Royse CF. Postoperative recovery and outcomes--what are we measuring and for whom?. Anaesthesia. 
2016; 71 Suppl 1: 72-7.

43. Sleney J, Christie N, Earthy S, Lyons RA, Kendrick D, Towner E. Improving recovery-Learning from patients' experiences after injury: a qualitative study. Injury. 2014; 45(1): 312-19.

44. Wisdom JP, Bruce K, Saedi GA, Weis T, Green CA. 'Stealing me from myself': identity and recovery in personal accounts of mental illness. Aust N Z J Psychiatry. 2008; 42(6): 489-95.

45. Dunn EC, Wewiorski NJ, Rogers ES. The meaning and importance of employment to people in recovery from serious mental illness: results of a qualitative study. Psychiatr Rehabil J. 2008; 32(1): 59-62.
46. Roda F, Agosti M, Merlo A, et al. Psychometric validation of the Italian Rehabilitation Complexity ScaleExtended version 13. PLoS One. 2017; 12(10): e0178453.

47. Safa A, Masoudi Alavi N, AbedzadehKalahroudi M. Predictive Factors of Dependency in Activities of Daily Living Following Limb Trauma in the Elderly. Trauma Mon. 2016; 21(5): e25091.

48. Hungerford C, Dowling M, Doyle K. Recovery Outcome Measures: Is There a Place for Culture, Attitudes, and Faith? Perspect Psychiatr Care. 2015; 51(3): 171-9. 